\title{
Extracellular vesicles: masters of intercellular communication and potential clinical interventions
}

\author{
Jonathan M. Pitt, ${ }^{1,2,3}$ Guido Kroemer, ${ }^{4,5,6,7,8,9,10}$ and Laurence Zitvogel $1,2,3,11,12$ \\ IInstitut de Cancérologie Gustave Roussy Cancer Campus (GRCC), Villejuif, France. ${ }^{2}$ INSERM U1015, Villejuif, France. ${ }^{3}$ Université Paris Sud, Université Paris-Saclay, Faculté de Médecine, Le Kremlin Bicêtre, \\ France. ${ }^{4}$ INSERM U848, Villejuif, France. ${ }^{5}$ Metabolomics Platform, CRCC, Villejuif, France. ${ }^{6}$ Equipe 11 Labellisée Ligue Contre le Cancer, Centre de Recherche des Cordeliers, INSERM U1138, Paris, France. \\ 7Pôle de Biologie, Hôpital Européen Georges Pompidou, Assistance Publique-Hôpitaux de Paris (AP-HP), Paris, France. ${ }^{8}$ Université Paris Descartes, Sorbonne Paris Cité, Paris, France. \\ 9Université Pierre et Marie Curie, Paris, France. ${ }^{10}$ Karolinska Institute, Department of Women's and Children's Health, Karolinska University Hospital, Stockholm, Sweden. \\ ${ }^{11}$ INSERM U932, Institut Curie, Paris Cedex 05, France. ${ }^{12}$ Center of Clinical Investigations in Biotherapies of Cancer (CICBT) 507, Villejuif, France.
}

\begin{abstract}
Intercellular signaling via extracellular vesicles (EVs) is an underappreciated modality of cell-cell crosstalk that enables cells to convey packages of complex instructions to specific recipient cells. EVs transmit these instructions through their cargoes of multiple proteins, nucleic acids, and specialized lipids, which are derived from their cells of origin and allow for combinatorial effects upon recipient cells. This Review series brings together the recent progress in our understanding of EV signaling in physiological and pathophysiological conditions, highlighting how certain EVs, particularly exosomes, can promote or regulate infections, host immune responses, development, and various diseases - notably cancer. Given the diverse nature of EVs and their abilities to profoundly modulate host cells, this series puts particular emphasis on the clinical applications of EVs as therapeutics and as diagnostic biomarkers.
\end{abstract}

\section{Introduction}

Cell-cell communication is an essential component in mammalian development and preservation of homeostasis, ensuring fast and efficient responses to alterations or threats within the environment surrounding host cells. Beyond classical signaling through cell-cell contact and soluble factors, such as cytokines, inflammatory mediators, metabolites, and hormones, such intercellular communication also occurs through cellular release of extracellular vesicles (EVs). This mode of communication has the potential to deliver a particularly diverse array of messages to EV-accepting cells at a level beyond that of soluble factor signaling, since EVs may carry a number of bioactive molecules, surface receptors, and genetic information (e.g., protein-coding mRNAs and regulatory microRNAs [miRNAs]) (1-4). These functional contents vary according to the cell type of origin and the particular physiological and pathological conditions in existence at the time of their packaging and secretion via EVs (5). With the added consideration that almost all cell types have been revealed to produce EVs $(5,6)$, it seems remarkable that EV signaling often remains a less considered mode of crosstalk and regulation between cells. Moreover, the progress made in our understanding of EV crosstalk between cells has highlighted how manipulation of EVs in vivo or of custom-designed, clinical-grade EVs could make efficient therapeutic interventions of the future.

EVs consist of a lipid bilayer membrane, containing various proteins and receptors, which envelopes a diverse array of protein, nucleic acid, chemical, and structural contents derived from the

Conflict of interest: The authors have declared that no conflict of interest exists. Reference information: / Clin Invest. 2016;126(4):1139-1143. doi:10.1172/JCI87316. cell of origin $(2,5,7,8)$. A number of different EV subpopulations have been described, and their classification is usually dependent upon their size and specific biogenesis (9). The best studied of these are exosomes (also sometimes called nanovesicles; size ranging from 30-100 $\mathrm{nm}$ ), generated by reverse budding of multivesicular bodies within cells before their secretion (10). Other notable EVs include microvesicles (with an approximate size of $200 \mathrm{~nm}$ ), which are directly shed from the plasma membrane of cells, and apoptotic bodies $(1-2 \mu \mathrm{m})(9,10)$. This latter subpopulation of EVs, which arises from cells undergoing apoptosis, is rapidly engulfed by phagocytic cells. Due to its rapid elimination, this population is less well characterized.

EVs interact with recipient cells, which may be local or considerably distant from the originating cell, through a process entailing ligand/receptor signaling at the recipient cell surface and/or the fusion of vesicle and cell plasma membranes. In most cases, this leads to EV uptake through endocytosis. During this process, EV membrane constituents can be delivered to the recipient cell membrane and EV cargoes can enter the recipient cell cytoplasm or nucleus, thereby contributing additional signaling molecules and pathways and potentially resulting in a diverse range of functional consequences in the EVrecipient cell $(6,9-11)$.

In this issue of the JCI, a distinguished group of leading experts in the EV field review exciting recent advances that uncover how EV signaling can profoundly modulate infections, host immune responses, and various diseases such as cancer. Moreover, this Review series places particular emphasis on the development of therapeutic applications for EVs, from their monitoring as diagnostic biomarkers to their direct clinical use as anticancer immunotherapies. 


\section{EVs as regulators of neuronal, immune,} and inflammatory processes

The complex degree of signaling mediated by EVs is utilized by many body systems under both physiological and pathophysiological conditions. One example is the nervous system, which requires a particularly advanced level of interactive exchanges among sensory and motor neurons, interneurons, and glia. Upon depolarization, cortical neurons in culture release EVs containing various neuronal cell adhesion proteins along with subunits of glutamate receptors, which bind to secondary neurons and modulate synaptic signaling, respectively $(12,13)$. Moreover, it has been postulated that neurons dispose of neurotransmitter receptors and miRNAs via EVs to regulate their excitability $(13,14)$. In this series, Zappulli and colleagues review the role of EVs in determining early neuronal development, synaptic strength, neuronal communication, and nerve regeneration (15). The authors also discuss the evidence for a contribution of EVs to glioblastoma and in neurodegenerative diseases, including Alzheimer's disease, Parkinson's disease, and prion diseases, which potentially involve EV-mediated spread of toxic or "infectious" misfolded proteins from neuron to neuron.

EV signaling has particular significance in the regulation of physiological CNS function and in remodeling following injury, with astrocytes, microglia, oligodendrocytes, and neurons communicating with one another through EV secretion (16). This EV signaling may extend beyond the CNS. In inflammatory settings, recent findings suggest that EVs derived from neural stem cells induce activation of proinflammatory cytokine signaling in immune cells, highlighting a potential role for EV-mediated crosstalk between the CNS and the immune system outside of the blood-brain barrier (17). EV communication also appears to be an important determinant of the outcome of CNS pathologies, such as brain ischemia. In this issue, Zhang et al. detail EV signaling within the CNS, emphasizing its involvement in mediating neurorestorative events following stroke and neural injury (18).

Much of the research into intercellular communication via EVs has focused on their ability to regulate the immune system. EVs from both immune and nonimmune cells have been shown to either suppress or stimulate adaptive and innate arms of immunity (17, 19-25), and these effects likely depend on the environmental context (e.g., EVs released in physiological conditions vs. those released during infection, which may carry microbial antigens) and the type of EV a particular immune cell encounters. These findings also suggest that engineered EVs that target immune cells may be used as novel therapeutic interventions for conditions such as autoimmune diseases $(19,26,27)$. Robbins et al. review EV regulation of chronic inflammatory and immune processes as part of this series, with predictions for how modified EVs might be used therapeutically (28). Possible future applications include using EVs derived from antigen-pulsed antigen-presenting cells (APCs) as vaccines (29) or modifying EV-shedding cells - and therefore derived EVs - to express immunosuppressive or immunostimulatory cytokines or surface molecules that affect the propagation of immune responses $(19,26)$.

Given the substantial involvement of EV signaling in immune responses, it is perhaps unsurprising that EVs also participate during infections with pathogenic microbes. Various pathogen products, such as pathogen-associated molecular patterns (PAMPs), may be transported from sources of infection via EVs, allowing delivery of antigens or agonists of innate immune receptors to bystander innate immune cells (30-32). These shed EVs may derive from pathogen-infected cells or directly from the pathogens themselves (33). EV carriage of PAMPs acts as an important means of immune system activation for host defense (34). Interestingly, a recent study has suggested that infected host cells may also use exosomes as a way to expel intracellular bacteria that are resistant to lysosomal degradation (35). However, several pathogens have evolved methods to hijack host EV signaling, enabling manipulation of their environment and evasion of immune responses to promote chronic infection $(23,36-38)$. Moreover, a number of different pathogens have been shown to modify the intracellular pathways responsible for exosome biogenesis, for example, by interfering with the assembly of endosomal sorting complex required for transport (ESCRT) machinery $(39,40)$. Schorey and Harding describe these processes in the context of viral, bacterial, parasitic, and fungal infections as part of this Review series (41), with discussion of how exosomes could be used as vaccines against pathogens and how EVs released during infections might be useful diagnostic markers for infectious diseases.

\section{EVs in cancer}

Tumorigenesis was formerly considered to be a largely cell-autonomous process, but is now known to be heavily influenced by communication of cancer cells with their surroundings $(42,43)$. EVs are one of the many methods by which cancer cells communicate with themselves, other cell types (e.g., immune cells), and the surrounding supportive structures constituting the tumor microenvironment (TME). A recent study using an elegant Cre-LoxP system to directly identify tumor cells that take up EVs in vivo revealed that EVs released by malignant tumor cells are taken up by less malignant tumor cells located within either the same or distant tumors. Through this process, the less malignant tumor cells that received these EVs began to display enhanced migratory behavior and metastatic behavior, supporting the key role of EV crosstalk in tumor progression and metastasis (44).

Signaling via exosomes is perhaps the most prominent form of EV signaling in the context of cancer and certainly the best studied. In a detailed overview, Kalluri presents the biology and complex functional roles of exosomes in the TME, tumor-associated angiogenesis, cancer development, and metastasis (45). Exosome production by tumor cells has been particularly portrayed as a cancer-promoting mechanism, enabling tumors to modulate and suppress antitumor immune responses. A specific "immunosuppressive content" within tumor-derived exosomes (Tex) likely dictates this evasion of immunosurveillance; however, Tex also carry tumor-associated antigens (TAAs) and costimulatory molecules that allow stimulation of potentially protective anticancer immune responses. Indeed, the potential for vaccination strategies utilizing Tex has been put forward (46). With both immunostimulatory and immunoinhibitory characteristics, the biological role of Tex has been a source of much debate. As part of this Review series, Whiteside describes how the biology of Tex and their cargoes can facilitate propagation of tumor-mediated immune suppression and/or immune stimulation. They also discuss their roles in tumor development and how Tex can interfere with cancer immunotherapies (47). 
It is becoming increasingly apparent that the mRNA and miRNA contents of exosomes may endow them with potent reprogramming capacity for manipulation of recipient cells (1, $4,48)$. Although originally thought to operate only within their cells of origin, a pivotal study by Valadi et al. showed that miRNA transported by exosomes is functional and mediates downstream effects on signaling pathways within recipient cells (1). Much recent evidence has revealed how miRNAs transferred by EVs from cancer cells may facilitate the initiation and progression of the hallmarks of cancer (49-52). In a timely Review, Kosaka et al. discuss the current knowledge regarding the contribution of EV-associated miRNAs in tumorigenesis, invasion, metastasis, and recurrence (53). The authors also discuss how miRNAs isolated from serum EVs have potential use in cancer diagnosis and monitoring of cancer treatment and a novel intervention whereby tumor-derived detrimental EVs may be removed from the circulation (53).

\section{Clinical applications for EVs}

EVs as biomarkers of disease. Given their significant presence in most if not all bodily fluids, which makes them easily and noninvasively accessible, EVs have been investigated as potential biomarkers for many diseases (54). Exosomes in particular have recently received much attention following the discovery of their potential role as a new noninvasive cancer biomarker. Mutations in KRAS and p53 are detectable using genomic DNA found in exosomes derived from pancreatic cancer cell lines and the sera of pancreatic cancer patients (3). Moreover, a recent study has shown how the cell-surface proteoglycan glypican-1, which is enriched on cancer cell-derived exosomes, can be used to distinguish patients with benign pancreatic disease from patients with early- and late-stage pancreatic cancers (55). Moreover, the detection of exosome-anchored glypican-1 was more reliable than existing assays for pancreatic tumor biomarkers at distinguishing patients with and without cancer. EV glypican-1 did not, however, reliably identify patients with breast cancer and did not distinguish between different breast cancer subtypes (55), suggesting that similar studies will be required to identify EV-related biomarkers that are specific for other cancer types. Prostate cancer, one of the most frequently diagnosed cancers $(56,57)$, is an example in which diagnostic biomarkers associated with prostatederived EVs, termed "prostasomes," could have a huge impact, since this could allow an unprecedented accurate determination of aggressive versus nonaggressive disease forms. In this Review series, Zijlstra and Stoorvogel propose that screening of proteins and RNA in immunoisolated prostasomes may serve to locate biomarkers for prostate cancer diagnosis, differentiation, prognosis, and epidemiology (58).

Clinical administration of EVs. The multifaceted nature of EVs and their contents underscores the advantage of using EVs for therapy, as they carry a repertoire of bioactive molecules with a combinatorial capacity that would be challenging to recapitulate by artificial means. Accordingly, much effort has been put forth into establishing protocols for EV purification from dedicated cell cultures and for assessment of EV purity (59-61); these efforts also include the design of protocols for production and characterization of clinical-grade exosome products $(62,63)$.
The assurance of accurately defined (or the production of highpurity) EV preparations is essential for the progression of the EV field, not only for clinical administration of exogenous EVs, but also in future preclinical studies, as this enables a greater certainty that an observed bioactivity results from a particular EV subpopulation. Put another way, impure or ill-characterized EV subpopulations lead to confusion as to which EV subtype is producing an observed effect. In a detailed Review in this series, Xu et al. describe the different EV subtypes and practical considerations regarding their isolation for therapeutic purposes (64). The authors also discuss the importance of working with highly purified EV populations and methods to ensure purification, such as the proteomic profiling of EVs.

As a direct consequence of these efforts in EV purification and characterization, EVs have made progress in clinical trials, most notably clinical preparations of exosomes derived from DCs as a form of immunotherapy for cancer (65-67). DCs play a central role in initiating antigen-specific immunity due to their role as the specialized APCs of the immune system (68). DC-derived exosomes (Dex) convey many of the immunostimulatory properties of DCs, but with significant advantages over direct DC-based therapies. In the final Review of this series, we describe these properties of Dex along with the mechanisms by which they interact with and stimulate immune responses. Furthermore, we detail the significant progress Dex immunotherapies have made in clinical oncology trials (69).

\section{Conclusions}

This Review series brings together information on the diverse implications of EV signaling in health and disease, how EVs may be utilized as diagnostic disease biomarkers and therapeutics, and how their inherent properties potentially provide benefits over existing clinical interventions. Moreover, the series highlights how this means of intercellular crosstalk can transfer an extraordinarily detailed level of information that may be precisely targeted to a given recipient cell type, with the great potential to be harnessed in novel biotechnological and therapeutic approaches of the future (e.g., EVs engineered to deliver drugs or toxins to select cell populations). A striking recurrent finding is the ability of EVs to convey molecular messages over significant distances in the body, an example being the long-distance regulation of metastasis afforded by miRNAs carried (and efficiently protected) within cancer cell-derived EVs (53).

Although the complexity of EVs naturally broadens their functional impact, at the same time, this makes study of their activity difficult. The overall effect on a cell following EV uptake not only results from the combination of the particular EV-transferred components (determined by the cell of origin and its status at the time of secretion), but must also take into account the fact that different EVs can have synergistic or opposing effects on the recipient cell. The dedicated studies necessary to address these complex questions, and indeed the future success of the EV field, fundamentally require improvements in characterization of EVs. A more accurate defining of EV surface membrane composition and EV cargoes through approaches such as proteomic analysis (70) coupled with RNA and/or DNA sequencing (71) may yield significant advances. Alongside this, and as mentioned above, further improvements in the purification of EV subpopulations will aid the clinical use of EVs as therapeutics, diagnostics, and prognostics (64). 
From a different perspective, continued basic research into the intracellular machineries responsible for the biogenesis of different EV subtypes may eventually give rise to novel approaches through which particular EVs from a certain cell type can be controlled, either at the genetic level or by a targeted drug. A standardized nomenclature for EVs across laboratories would also benefit the field. Indeed, many investigators classify small EVs as "exosomes" even when such subpopulations have not been shown to originate from intracellular endosomes (i.e., in line with the original identifications of exosome biogenesis ref. 72; these issues are discussed in refs. 54, 73). Similarly, standardization in $\mathrm{EV}$ quantification, isolation, storage, and functional potency assays is also called for, as such assays are essential for the future clinical testing of EVs (e.g., in the administration of clinical exosome vaccine products) (64).

Based on the promising findings across the EV field thus far, it is likely that the next decade will see a significant increase in clinical studies harnessing EVs as therapies or diagnostics. We hope that new strategies and technological advances that beneficially manipulate EVs will also mature, a possible example being an approach to effectively removing untoward EVs, such as the Tex and other cancer-derived EVs that promote immunosuppression in cancer, from the circulation.

\section{Acknowledgments}

We thank Association de Recherche Contre le Cancer for supporting J.M. Pitt. L. Zitvogel received a special prize from the Swiss Bridge Foundation and the Swiss Institute for Experimental Cancer Research (ISREC). G. Kroemer and L. Zitvogel were supported by the Ligue Nationale Contre le Cancer (Equipes Labellisées), the Agence Nationale Pour la Recherche (ANR AUTOPH, ANR Emergence), the European Commission (ArtForce), a European Research Council Advanced Investigator grant (to G. Kroemer), the Fondation pour la Recherche Médicale (FRM), the Institut National du Cancer (INCa), the Fondation de France, the Cancéropôle Ile-de-France, the Fondation Bettencourt-Schueller, the Swiss Bridge Foundation, the LabEx Immuno-Oncology, SIRIC Stratified Oncology Cell DNA Repair and Tumor Immune Elimination (SOCRATE); SIRIC Cancer Research and Personalized Medicine (CARPEM), and the Paris Alliance of Cancer Research Institutes (PACRI). L. Zitvogel and G. Kroemer are sponsored by the Association Pour la Recherche Contre le Cancer (PGA120140200851).

Address correspondence to: Laurence Zitvogel, Gustave Roussy Cancer Campus, 114 rue Edouard Vaillant, 94805 VILLEJUIF Cedex, France. Phone: 33.1.42.11.26.79; Phone: 33.1.42.11.50.41; E-mail: laurence.zitvogel@gustaveroussy.fr.
1. Valadi H, Ekstrom K, Bossios A, Sjostrand M, Lee JJ, Lotvall JO. Exosome-mediated transfer of mRNAs and microRNAs is a novel mechanism of genetic exchange between cells. Nat Cell Biol. 2007;9(6):654-659.

2. Thery C, Zitvogel L, Amigorena S. Exosomes: composition, biogenesis and function. Nat Rev Immunol. 2002;2(8):569-579.

3. Kahlert C, et al. Identification of double-stranded genomic DNA spanning all chromosomes with mutated KRAS and p53 DNA in the serum exosomes of patients with pancreatic cancer. J Biol Chem. 2014;289(7):3869-3875.

4. Ratajczak J, et al. Embryonic stem cell-derived microvesicles reprogram hematopoietic progenitors: evidence for horizontal transfer of mRNA and protein delivery. Leukemia. 2006;20(5):847-856.

5. Raposo G, Stoorvogel W. Extracellular vesicles: exosomes, microvesicles, and friends. JCell Biol. 2013;200(4):373-383

6. Robbins PD, Morelli AE. Regulation of immune responses by extracellular vesicles. Nat Rev Immunol. 2014;14(3):195-208.

7. Thery C, et al. Proteomic analysis of dendritic cell-derived exosomes: a secreted subcellular compartment distinct from apoptotic vesicles. JImmunol. 2001;166(12):7309-7318.

8 . Thery C, et al. Molecular characterization of dendritic cell-derived exosomes. Selective accumulation of the heat shock protein hsc73. J Cell Biol. 1999;147(3):599-610.

9. Thery C, Ostrowski M, Segura E. Membrane vesicles as conveyors of immune responses. Nat Rev Immunol. $2009 ; 9(8): 581-593$.

10. Harding CV, Heuser JE, Stahl PD. Exosomes: looking back three decades and into the future. JCell Biol. 2013;200(4):367-371.

11. Yang C, Robbins PD. The roles of tumor-derived exosomes in cancer pathogenesis. Clin Dev Immunol. 2011;2011:842849.

12. Chivet M, Hemming F, Pernet-Gallay K, Fraboulet S, Sadoul R. Emerging role of neuronal exosomes in the central nervous system. Front Physiol. 2012;3:145.

13. Faure J, et al. Exosomes are released by cultured cortical neurones. Mol Cell Neurosci. 2006;31(4):642-648.

14. Goldie BJ, et al. Activity-associated miRNA are packaged in Map1b-enriched exosomes released from depolarized neurons. Nucleic Acids Res. 2014;42(14):9195-9208.

15. Zappulli V, Friis KP, Fitzpatrick Z, Maguire CA, Breakefield XO. Extracellular vesicles and intercellular communication within the nervous system. J Clin Invest. 2016;126(4):1198-1207.

16. Frühbeis C, Fröhlich D, Kuo WP, Krämer-Albers EM. Extracellular vesicles as mediators of neuron-glia communication. Front Cell Neurosci. 2013;7:182.

17. Cossetti C, et al. Extracellular vesicles from neural stem cells transfer IFN- $\gamma$ via Ifngr1 to activate Stat1 signaling in target cells. Mol Cell. 2014;56(2):193-204.

18. Zhang ZG, Chopp M. Exosomes in stroke pathogenesis and therapy. JClin Invest. 2016;126(4):1190-1197.

19. Kim SH, et al. Exosomes derived from IL10-treated dendritic cells can suppress inflammation and collagen-induced arthritis. J Immunol. 2005;174(10):6440-6448.

20. Montecalvo A, et al. Exosomes as a short-range mechanism to spread alloantigen between dendritic cells during $\mathrm{T}$ cell allorecognition. J Immunol. 2008;180(5):3081-3090.

21. Segura E, Amigorena S, Thery C. Mature dendritic cells secrete exosomes with strong ability to induce antigen-specific effector immune responses. Blood Cells Mol Dis. 2005;35(2):89-93.

22. Segura E, et al. ICAM-1 on exosomes from mature dendritic cells is critical for efficient naive T-cell priming. Blood. 2005;106(1):216-223.

23. Singh PP, LeMaire C, Tan JC, Zeng E, Schorey JS. Exosomes released from M. tuberculosis infected cells can suppress IFN- $\gamma$ mediated activation of naive macrophages. PLoS One. 2011;6(4):e18564.

24. Thery C, Duban L, Segura E, Veron P, Lantz O, Amigorena S. Indirect activation of naive $\mathrm{CD} 4^{+}$ T cells by dendritic cell-derived exosomes. Nat Immunol. 2002;3(12):1156-1162.

25 . Viaud S, et al. Dendritic cell-derived exosomes promote natural killer cell activation and proliferation: a role for NKG2D ligands and IL-15R $\alpha$. PLoS One. 2009;4(3):e4942.

26. $\mathrm{Kim} \mathrm{SH}$, et al. Exosomes derived from genetically modified DC expressing FasL are antiinflammatory and immunosuppressive. Mol Ther. 2006;13(2):289-300

27. $\mathrm{Bu} \mathrm{N}$, et al. Immature dendritic cell exosomes suppress experimental autoimmune myasthenia gravis. J Neuroimmunol. 2015;285:71-75.

28. Robbins PD, Dorronsoro A, Booker CN. Regulation of chronic inflammatory and immune processes by extracellular vesicles. JClin Invest. 2016;126(4):1173-1180.

29. Beauvillain C, Ruiz S, Guiton R, Bout D, DimierPoisson I. A vaccine based on exosomes secreted by a dendritic cell line confers protection against $\mathrm{T}$. gondii infection in syngeneic and allogeneic mice. Microbes Infect . 2007;9(14-15):1614-1622.

30. Aline F, Bout D, Amigorena S, Roingeard P, Dimier-Poisson I. Toxoplasma gondii antigenpulsed-dendritic cell-derived exosomes induce a protective immune response against $\mathrm{T}$. gondii 
infection. Infect Immun. 2004;72(7):4127-4137.

31. Giri PK, Schorey JS. Exosomes derived from M. Bovis BCG infected macrophages activate antigen-specific $\mathrm{CD} 4^{+}$and $\mathrm{CD} 8^{+} \mathrm{T}$ cells in vitro and in vivo. PLoS One. 2008;3(6):e2461.

32. Kuate S, Cinatl J, Doerr HW, Uberla K. Exosomal vaccines containing the $S$ protein of the SARS coronavirus induce high levels of neutralizing antibodies. Virology. 2007;362(1):26-37.

33. Kulp A, Kuehn MJ. Biological functions and biogenesis of secreted bacterial outer membrane vesicles. Annu Rev Microbiol. 2010;64:163-184.

34. Dreux M, et al. Short-range exosomal transfer of viral RNA from infected cells to plasmacytoid dendritic cells triggers innate immunity. Cell Host Microbe. 2012;12(4):558-570.

35. Miao Y, Li G, Zhang X, Xu H, Abraham SN. A TRP channel senses lysosome neutralization by pathogens to trigger their expulsion. Cell. 2015;161(6):1306-1319.

36. Gambarte Tudela J, et al. The late endocytic Rab39a GTPase regulates the interaction between multivesicular bodies and chlamydial inclusions. J Cell Sci. 2015;128(16):3068-3081.

37. Meckes DG Jr. Exosomal communication goes viral. J Virol. 2015;89(10):5200-5203.

38. Feng Z, et al. A pathogenic picornavirus acquires an envelope by hijacking cellular membranes. Nature. 2013;496(7445):367-371.

39. Boonyaratanakornkit J, Schomacker H, Collins P, Schmidt A. Alix serves as an adaptor that allows human parainfluenza virus type 1 to interact with the host cell ESCRT system. PLoS One. 2013;8(3):e59462.

40. Mehra A, et al. Mycobacterium tuberculosis type VII secreted effector EsxH targets host ESCRT to impair trafficking. PLoS Pathog. 2013;9(10):e1003734.

41. Schorey JS, Harding CV. Extracellular vesicles and infectious diseases: new complexity to an old story. J Clin Invest. 2016;126(4):1181-1189.

42. Chaffer CL, Weinberg RA. A perspective on cancer cell metastasis. Science. 2011;331(6024):1559-1564.

43. Marusyk A, Tabassum DP, Altrock PM, Almendro V, Michor F, Polyak K. Non-cell-autonomous driving of tumour growth supports sub-clonal heterogeneity. Nature. 2014;514(7520):54-58.

44. Zomer A, et al. In vivo imaging reveals extracellular vesicle-mediated phenocopying of metastatic behavior. Cell. 2015;161(5):1046-1057.

45. Kalluri R. The biology and function of exosomes in cancer. J Clin Invest. 2016;126(4):1208-1215.
46. Andre F, et al. Tumor-derived exosomes: a new source of tumor rejection antigens. Vaccine. 2002;20(suppl 4):A28-A31.

47. Whiteside TL. Exosomes and tumor-mediated immune suppression. J Clin Invest. 2016;126(4):1216-1223.

48. Zhang J, et al. Exosome and exosomal microRNA: trafficking, sorting, and function. Genomics Proteomics Bioinformatics. 2015;13(1):17-24.

49. Le MT, et al. miR-200-containing extracellular vesicles promote breast cancer cell metastasis. J Clin Invest. 2014;124(12):5109-5128.

50. Ono M, et al. Exosomes from bone marrow mesenchymal stem cells contain a microRNA that promotes dormancy in metastatic breast cancer cells. Sci Signal. 2014;7(332):ra63.

51. Skog J, et al. Carter BS, Krichevsky AM, and Breakefield XO. Glioblastoma microvesicles transport RNA and proteins that promote tumour growth and provide diagnostic biomarkers. Nat Cell Biol. 2008;10(12):1470-1476.

52. Melo SA, et al. Cancer exosomes perform cellindependent microRNA biogenesis and promote tumorigenesis. Cancer Cell. 2014;26(5):707-721.

53. Kosaka N, Yoshioka Y, Fujita Y, Ochiya T. Versatile roles of extracellular vesicles in cancer. J Clin Invest. 2016;126(4):1163-1172.

54. Thery C. Cancer: Diagnosis by extracellular vesicles. Nature. 2015;523(7559):161-162.

55. Melo SA, et al. Glypican-1 identifies cancer exosomes and detects early pancreatic cancer. Nature. 2015;523(7559):177-182.

56 . Ferlay J, et al. Cancer incidence and mortality worldwide: sources, methods and major patterns in GLOBOCAN 2012. Int J Cancer. 2015;136(5):E359-E386.

57. Ferlay J, et al. Cancer incidence and mortality patterns in Europe: estimates for 40 countries in 2012. Eur J Cancer. 2013;49(6):1374-1403.

58. Zijlstra C, Stoorvogel W. Prostasomes as a source of diagnostic biomarkers for prostate cancer. J Clin Invest. 2016;126(4):1144-1151.

59. Lobb RJ, et al. Optimized exosome isolation protocol for cell culture supernatant and human plasma. J Extracell Vesicles. 2015;4:27031.

60. Greening DW, Xu R, Ji H, Tauro BJ, Simpson RJ. A protocol for exosome isolation and characterization: evaluation of ultracentrifugation, density-gradient separation, and immunoaffinity capture methods. Methods Mol Biol. 2015;1295:179-209.

61. Thery C, Amigorena S, Raposo G, Clayton A. Iso- lation and characterization of exosomes from cell culture supernatants and biological fluids. Curr Protoc Cell Biol. 2006; Chapter 3:Unit 3.22.

62. Viaud S, et al. Updated technology to produce highly immunogenic dendritic cell-derived exosomes of clinical grade: a critical role of interferon-gamma. J Immunother. 2011;34(1):65-75.

63. Lamparski HG, et al. Production and characterization of clinical grade exosomes derived from dendritic cells. J Immunol Methods. 2002;270(2):211-226.

64. Xu R, Greening DW, Zhu H-J, Takahashi N, Simpson RJ. Extracellular vesicle isolation and characterization: toward clinical application.

JClin Invest. 2016;126(4):1152-1162.

65. Besse B, et al. Dendritic cell-derived exosomes as maintenance immunotherapy after first line chemotherapy in NSCLC [published online August 12, 2015]. Oncoimmunology. doi:10.1080/216240 2X.2015.1071008.

66. Escudier B, et al. Vaccination of metastatic melanoma patients with autologous dendritic cell (DC) derived-exosomes: results of the first phase I clinical trial. J Transl Med. 2005;3(1):10.

67. Morse MA, et al. A phase I study of dexosome immunotherapy in patients with advanced nonsmall cell lung cancer. J Transl Med. 2005;3(1):9.

68. Steinman RM. Decisions about dendritic cells: past, present, and future. Annu Rev Immunol. 2012;30:1-22.

69. Pitt JM, et al. Dendritic cell-derived exosomes for cancer therapy. J Clin Invest. 2016;126(4):1224-1232.

70. Xu R, Greening DW, Rai A, Ji H, Simpson RJ. Highly-purified exosomes and shed microvesicles isolated from the human colon cancer cell line LIM1863 by sequential centrifugal ultrafiltration are biochemically and functionally distinct. Methods. 2015;87:11-25.

71. Ji H, et al. Deep sequencing of RNA from three different extracellular vesicle (EV) subtypes released from the human LIM1863 colon cancer cell line uncovers distinct miRNA-enrichment signatures. PLoS One. 2014;9(10):e110314.

72. Johnstone RM, Adam M, Hammond JR, Orr L, Turbide C. Vesicle formation during reticulocyte maturation. Association of plasma membrane activities with released vesicles (exosomes). J Biol Chem. 1987;262(19):9412-9420.

73. Gould SJ, Raposo G. As we wait: coping with an imperfect nomenclature for extracellular vesicles. JExtracell Vesicles. 2013;2. 